\title{
Optical alignment of the Solar Orbiter EUI flight instrument
}

A. Mazzolia, J.-P. Halain ${ }^{b}$, F. Auchèrec ${ }^{c}$, J. Barbayc ${ }^{c}$, S. Meining ${ }^{d}$, A. Philipponc, G. Morinaudc, S. Roose $^{a}$, M.-L. Hellina, L. Jacques'a, U. Schühled, C. Dumesnilc, R. Merciere, E. Renotte ${ }^{f}$, P. Rochus ${ }^{a}$
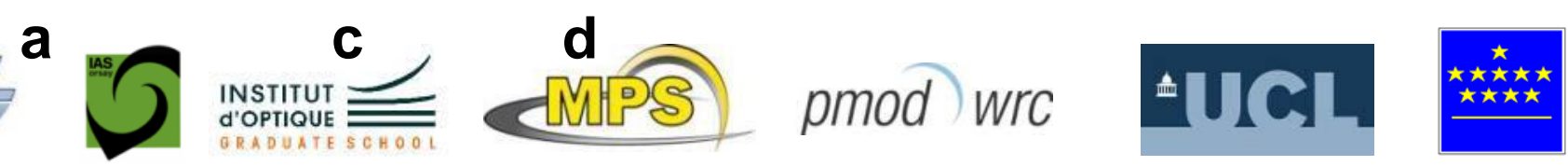

bESTEC - European Space Research and Technology Center, 'Laboratoire Charles Fabry, Institut d'Optique Graduate School

${ }^{f}$ AMOS, Liege Science Park, 2 Rue des Chasseurs Ardennais, 4031 Angleur, Belgium. 


\section{Outlook}

- EUI instrument \& challenges

- HRI EUV

- Alignment methodology

- Alignment tools

- HRI EUV alignment

- HRI Ly alignment

- FSI Alignment

- Co alignment

- Conclusions 


\section{Solar Orbiter is a new unique solar mission}

Innovative and challenging due to

- its orbit (get close to the sun at 0,28 AU, closer than Mercury and out of the plane of the ecliptic at $25^{\circ}$, then $34^{\circ}$ in the extended mission)

- its payload: an original combination of 10 instruments: 4 in situ and 6 remote sensing.
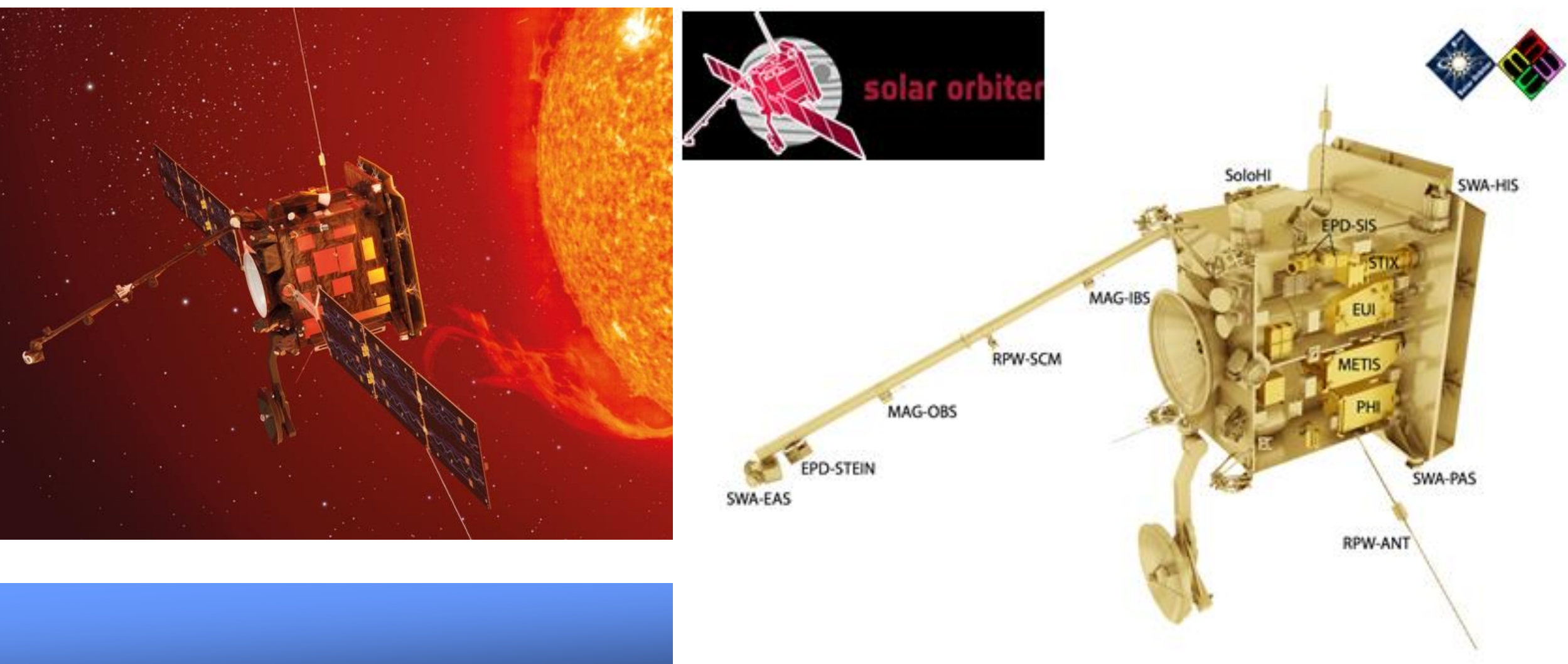


\section{EUI instrument}

\section{- EUI instrument}

- Three channels telescope

\begin{tabular}{|l|l|l|}
\hline Channel & Parameter & Values \\
\hline \multirow{4}{*}{ FSI } & Passband & $17.4 \mathrm{~nm} \& 30.4 \mathrm{~nm}$ \\
& FOV & $3.8 \mathrm{arcdeg}$ \\
& Resolution (2 px) & 9 arcsec \\
& Cadence & $600 \mathrm{~s}$ \\
\hline \multirow{4}{*}{ HRI $_{\text {EUV }}$} & Passband & $17.4 \mathrm{~nm}$ \\
& FOV & $0.28 \operatorname{arcdeg}$ \\
& Resolution $(2 \mathrm{px})$ & $1 \operatorname{arcsec}$ \\
& Cadence & $\geq 1 \mathrm{~s}$ \\
\hline \multirow{3}{*}{ HRI $_{\text {Lya }}$} & Passband & $121.6 \mathrm{~nm}$ \\
& FOV & $0.28 \mathrm{arcdeg}$ \\
& Resolution $(2 \mathrm{px})$ & $1 \mathrm{arcsec}$ \\
& Cadence & $\leq 1 \mathrm{~s}$ \\
\hline
\end{tabular}

\section{$174 \AA$ (Fe X) and $304 \AA$ ( He II);}

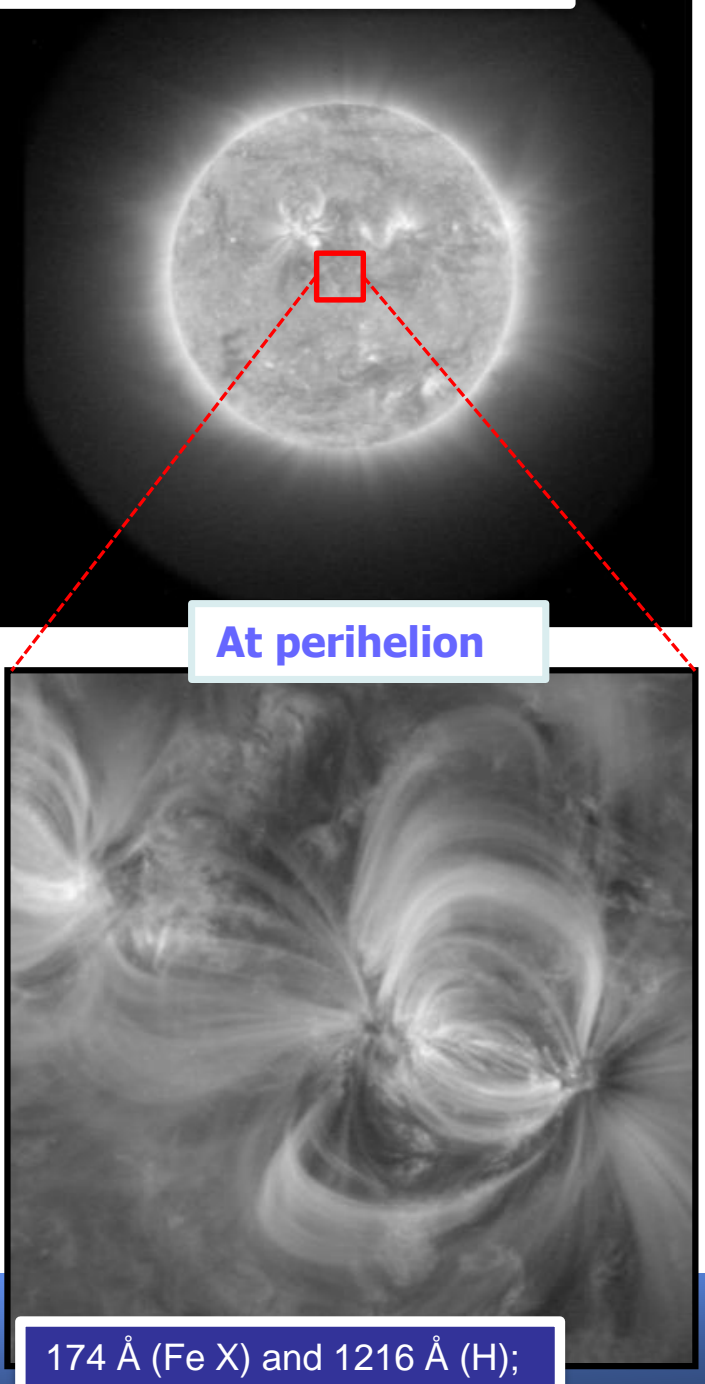




\section{EUI challenges}

\section{- Two units}

$-25 \mathrm{~kg}(\mathrm{OBS}=17 \mathrm{~kg}, \mathrm{CEB}=8 \mathrm{~kg})$

$-30.5 \mathrm{~W}$ average (35 W peak)

OBS (optical bench system)

Specific to the mission:

-Thermal environment

- Compactness of the suite of 3 telescopes

-Very high efficiency of detecting chain

-Very limited telemetry

CEB (common electronic box)

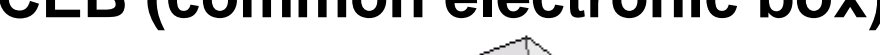




\section{HRI EUI design}

\section{The HRI $\mathrm{EUV}_{\text {channel is based compact two }}$ mirrors off-axis design}

TABLE I. HRI EUV CHANNEL REQUIREMENTS

\begin{tabular}{|c|c|}
\hline Parameter & Requirement \\
\hline Field of view & 1000 arcsec square \\
\hline Entrance pupil & $47.4 \mathrm{~mm}$ diameter \\
\hline Detector size & $\begin{array}{c}2048 \times 2048 \text { pixels } \\
10 \mu \mathrm{m} \text { pixel pitch }\end{array}$ \\
\hline Spot size & 1 arcsec (i.e. 2 pixels) \\
\hline $\begin{array}{c}\text { Incident angles on } \\
\text { mirrors }\end{array}$ & $<6$ arcdeg \\
\hline Spectral range & $174 \AA$ \\
\hline Dimensions & $<820 \mathrm{~mm}$ length \\
$<120 \mathrm{~mm}$ width
\end{tabular}

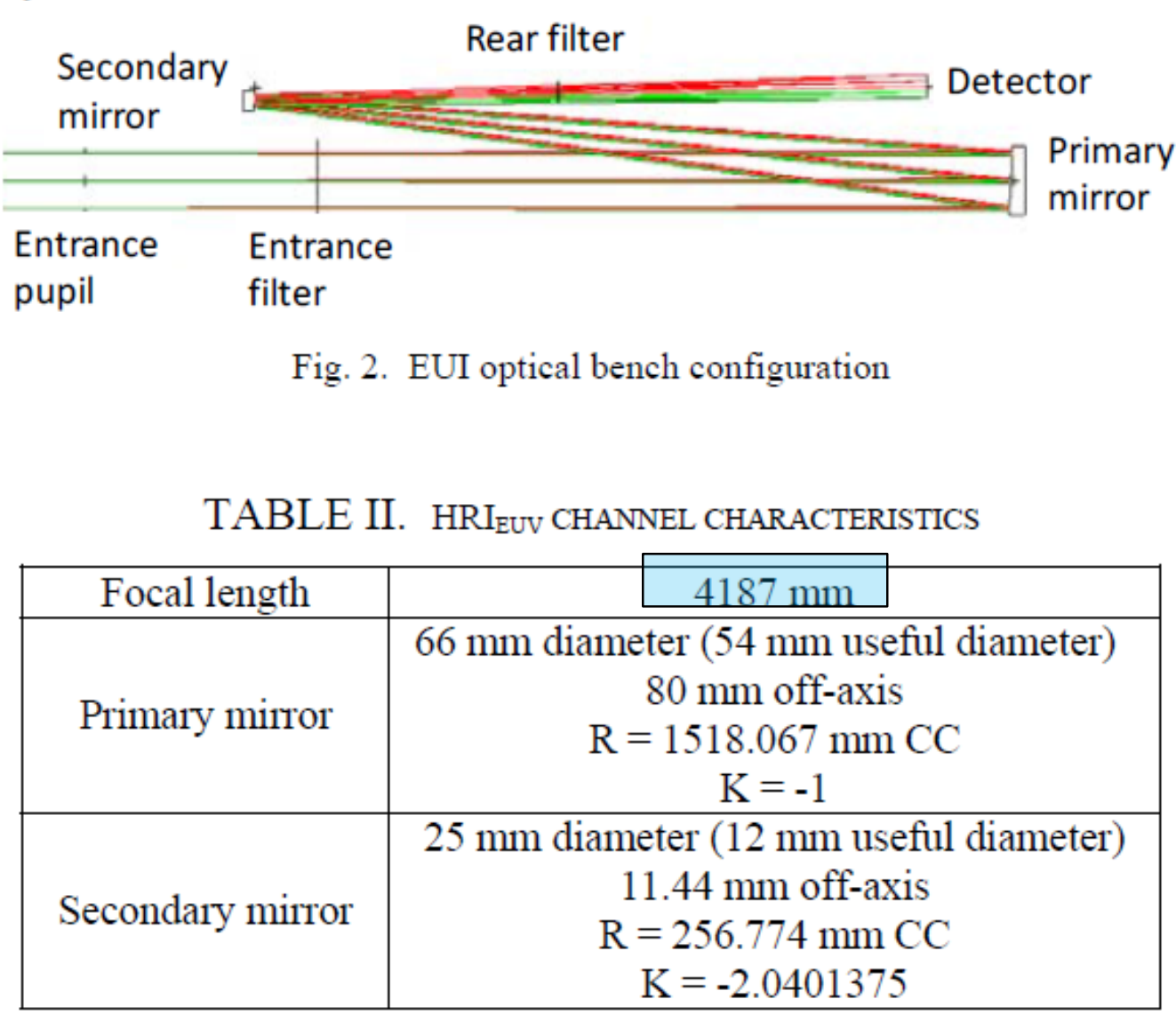




\section{ALIGNMENT TOOLS}

- Theodolites (T6000, T5100, T3000, T2 ...)

- A large flat transfer mirror

- An interferometer with an alignment plate

- A 3D measurement arm
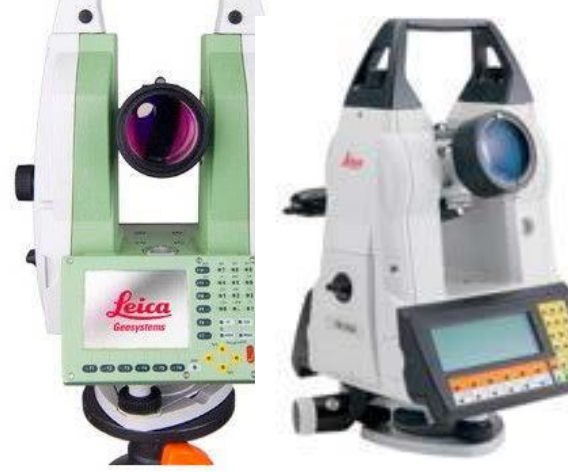

- Specific alignment tools have been designed for the HRI EUV M1 and M2 mirrors to adjust their roll and simplify the shimming

- Dummy camera
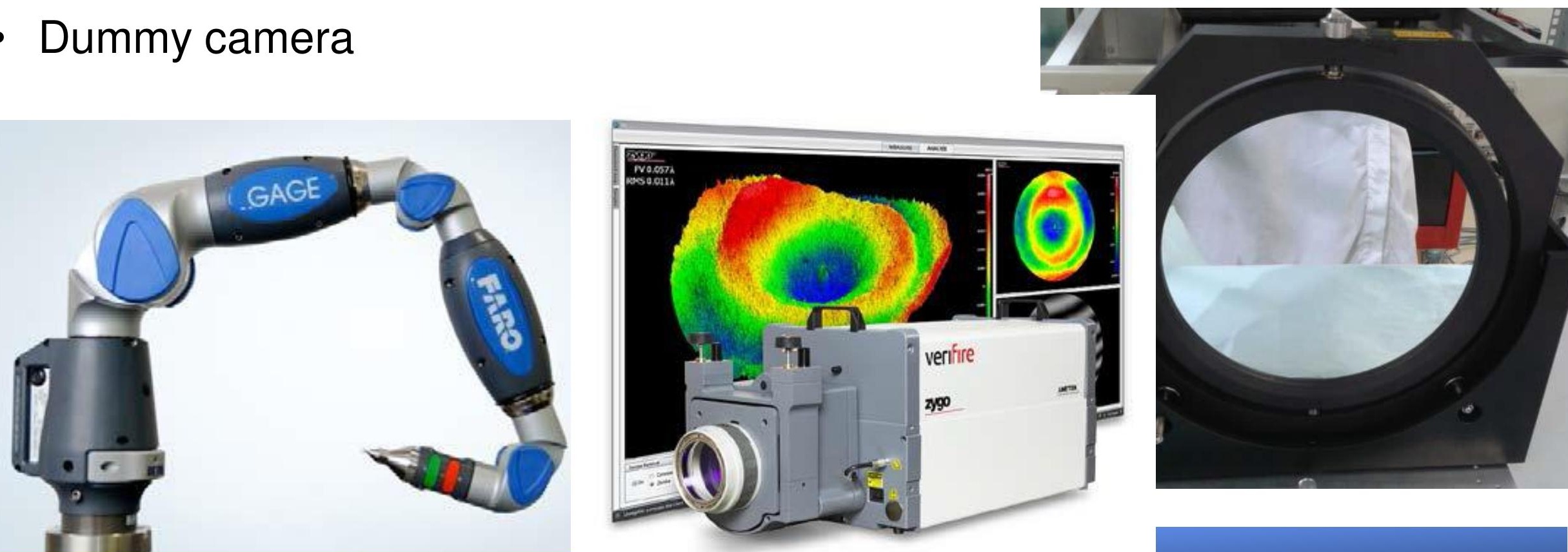


\section{ALIGNMENT METHODOLOGY}

1. Measure mirror characteristics after manufacturing (WFE, vertex position, curvature...).

2. Introduce the measured values in the optical model

3. Optimize M2 and M1 mirror pos/orient mirror to min the spots size 4. Set M1 mirror orientation w.r.t. the optical bench reference cube, i.e. instrument optical axis (using theodolite)

5. Set the M2 on the instrument bench at the position and orientation as provided by the updated optical model (mechanical positioning)

6. Optimize the WFE using M2 mirror position/orientation (interferometric measurement)

7. Set the detector in the real FPA based on dummy FPA settings,

8. Use the real FPA to cross-check alignment (using collimated light beam ) 


\section{OPTICAL ALIGNMENT ACTIVITIES}

$H R I_{E U V}$ mirrors 1) M1 orientation w.r.t. the reference cube (which defines the URAF)

2) position M1 \& M2 w.r.t. the theoretical position predicted by the optical model. Adjustment by shimming

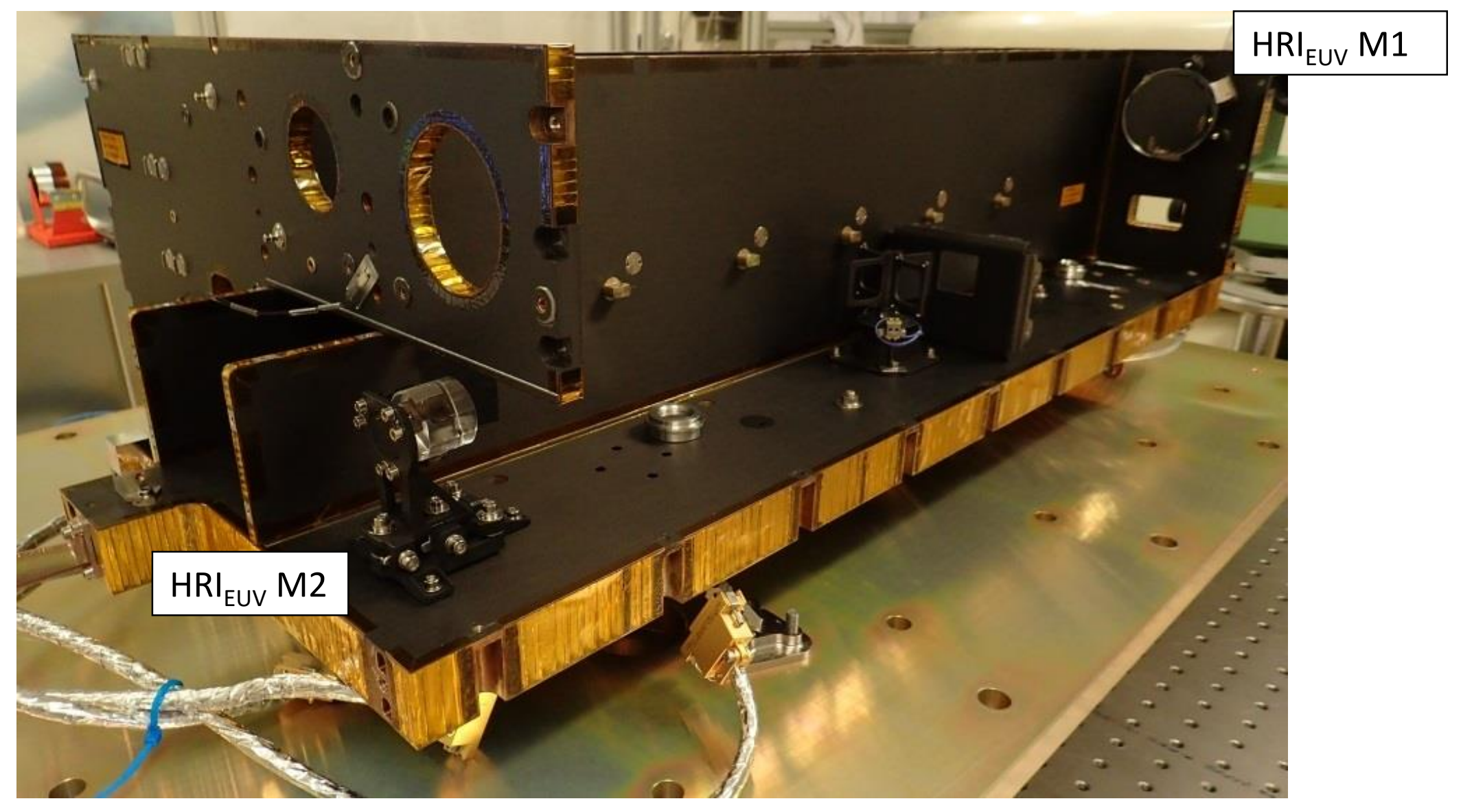




\section{$C S L$ HRIEUV front side interferometric method}

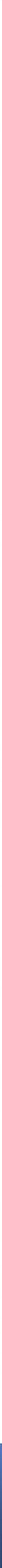




\section{method
mRIEUv interferometric measurement - back-side}

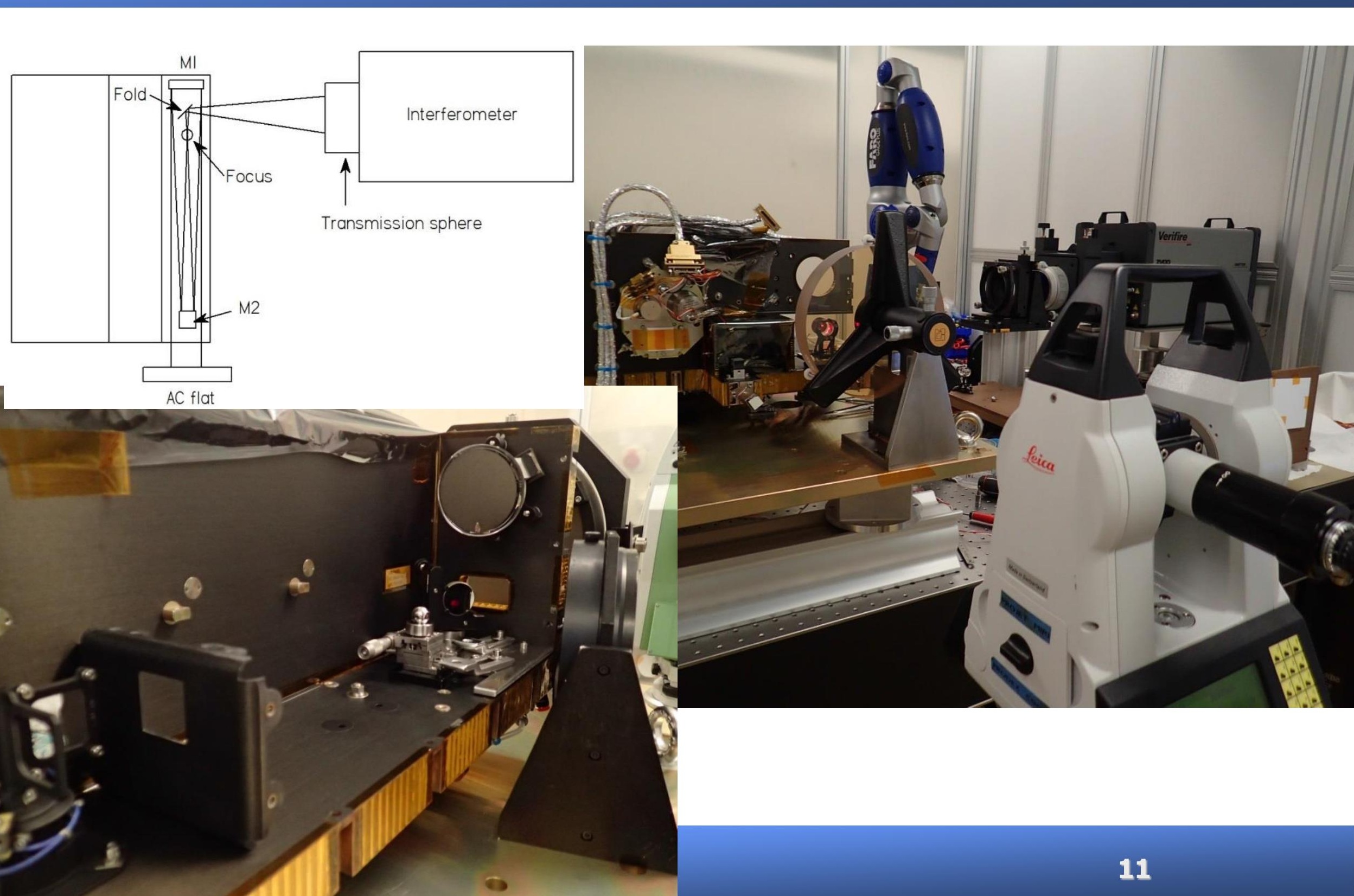




\section{HRI $I_{E U V}$ final alignment WFE}

WFE $\rightarrow$ residual focus term $\rightarrow$ required displacement of M2 focus is calculated and applied with shims.

Final WFE of the telescope

- PV 0.057 waves ( $\Leftrightarrow 36 \mathrm{~nm}$ )

- RMS amplitudes 0.009 waves $(\Leftrightarrow 5.7 \mathrm{~nm})<\operatorname{spec} 0.01$ wave

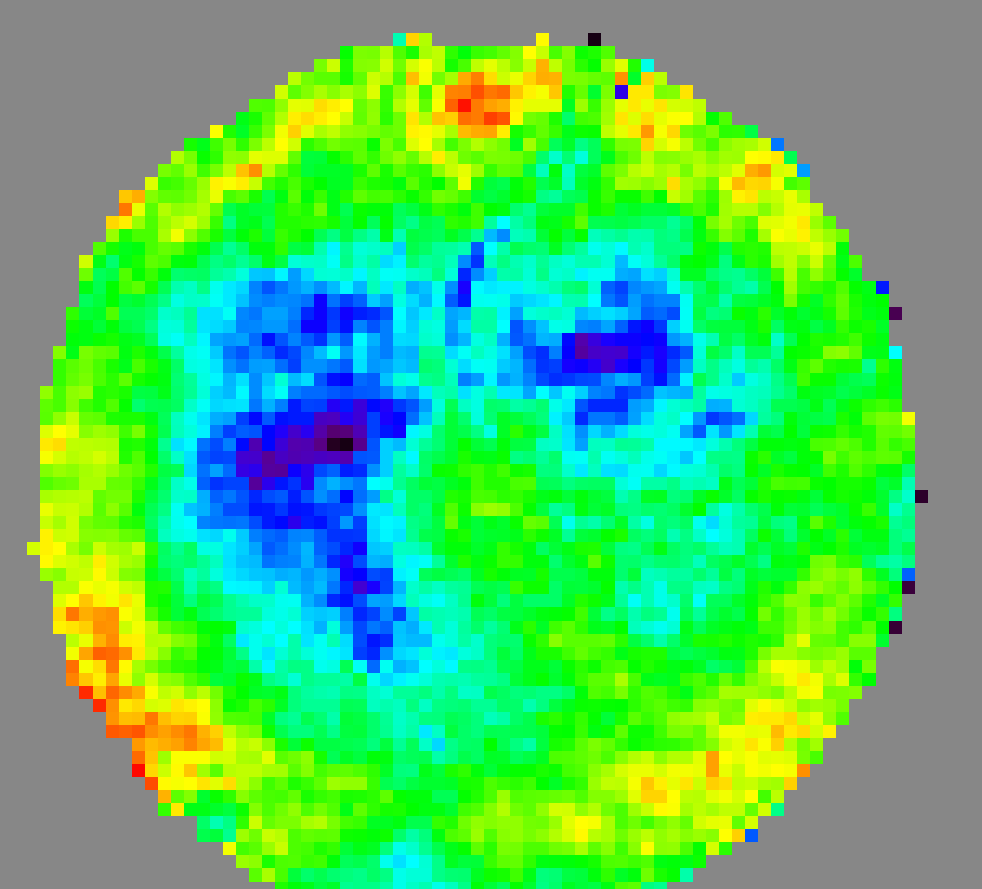

Final alignment, the flat orientation has been corrected by 16 arcsec in horizontal and 11 arcsec in vertical to cancel the tilt in the WFE.

$\rightarrow$ the measured WFE does not exactly correspond to the central part of the Field of View (FOV) of the instrument but it is very close. 


\section{Detector positioning}

The residual focus term $+0.12 \mathrm{~mm}$ to be added for best focus for the whole FOV

$\rightarrow$ A correction of $0.5 \mathrm{~mm}$ on the detector support

$\rightarrow$ Residual focus enlarges of $+/-1 \mu \mathrm{m}$ the image spots

(RMS diameter is $7 \mu \mathrm{m}$ without residual focus).

HRIEUV detector at final position

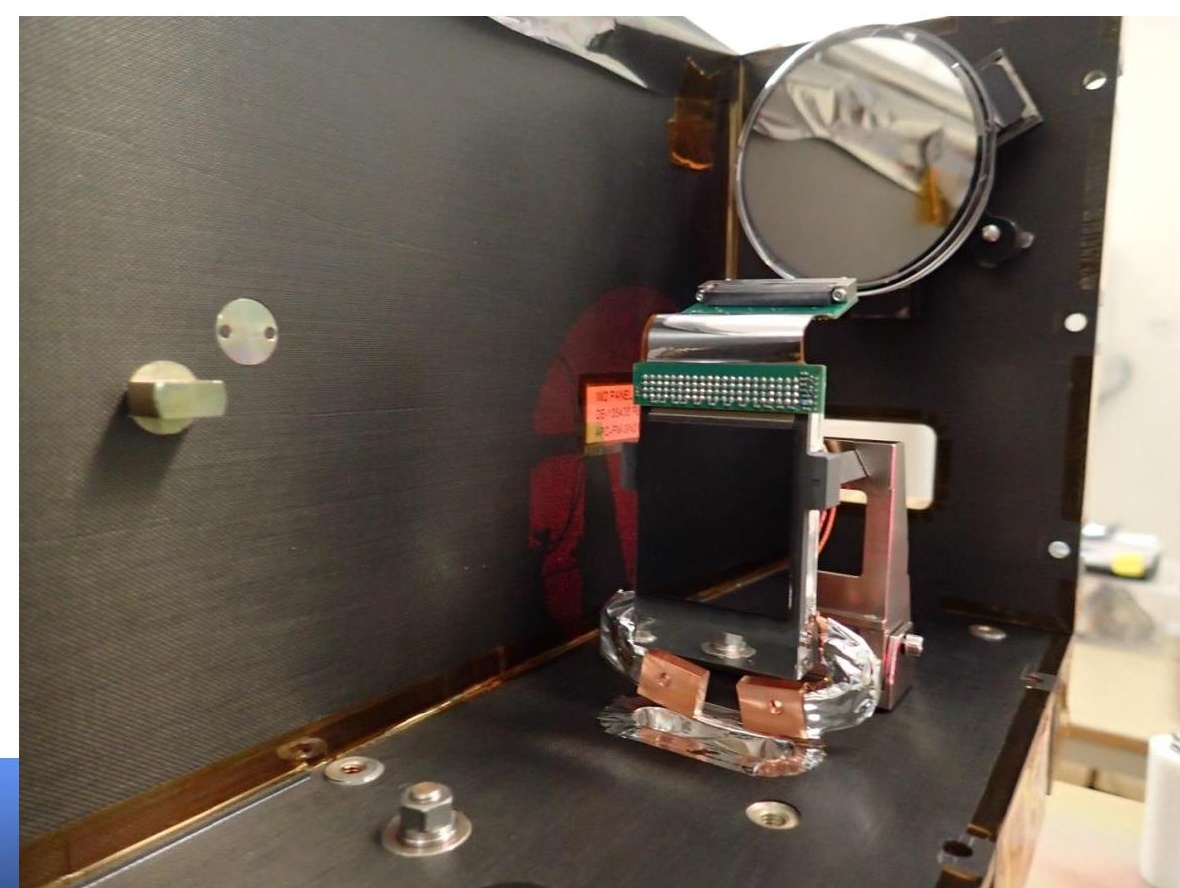




\section{HRI $_{L Y-a}$ CHANNEL ALIGNMENT}

The interferometric alignment of the FM mirrors has been performed on a dummy bench.

The mirrors have then been moved on the FM bench and the alignment has been verified geometrically (orientations and positions of the mirrors) using 3D arm and theodolites. 


\section{FSI Channel Alignment}

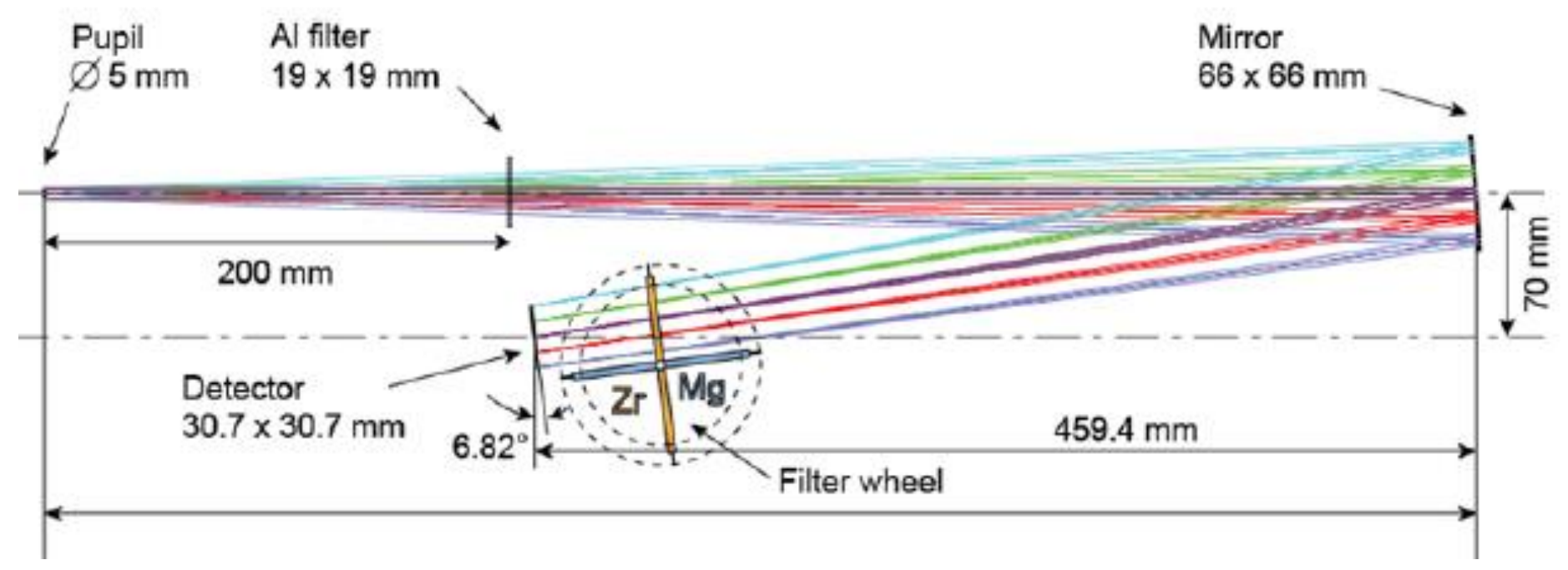

Alignment steps:

1. Alignment of the mirror with regards to the mechanical axis

2. Alignment of the dummy FPA on axis and for different FOV using the interferometer

3. Integration of FM FPA

4. Integration of FSI filter wheel

5. Alignment of the filter wheel with regards to the optical axis

6. Integration of the FM filters on the wheel. 


\section{FSI interferometric measurement}

The fine tuning of the alignment of the FPA has been performed using a dummy FPA. The dummy FPA consists of an array of pinhole and spherical mirror centered on each FOV.

Alignment on the central FOV + verification for 3 other FOVs.

At the end of the process, the measured distance between the FPA and the mirror is $20 \mu \mathrm{m}$ from the theoretical value $\rightarrow$ The FSI channel within specified tolerances.

\section{FSI aligned telescope, with camera}

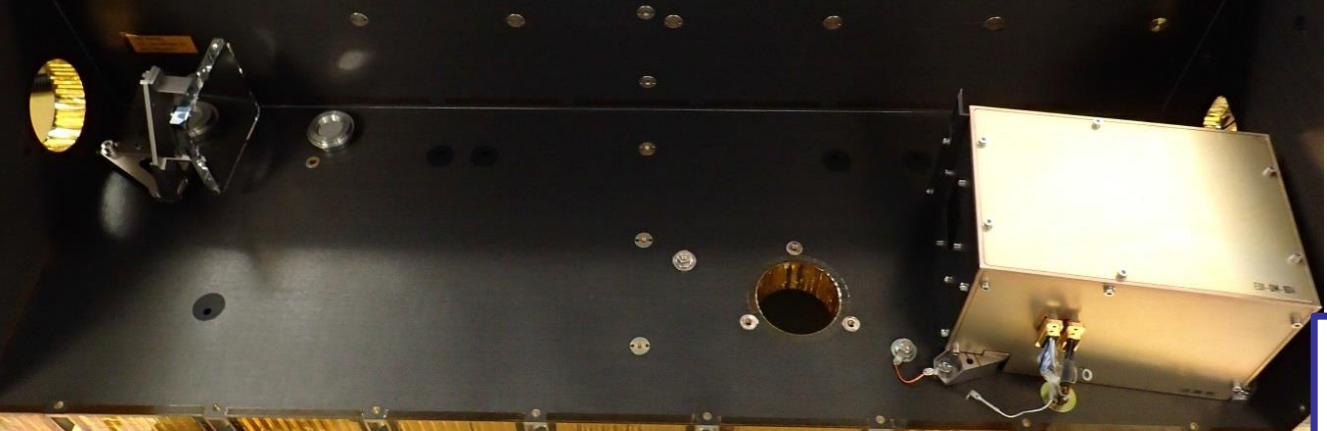

\begin{tabular}{|l|l}
\hline parameter & value \\
\hline$\Delta \theta$ Y (horizontal) & $+33^{\prime \prime}$ \\
\hline$\Delta \theta X$ (vertical) & $-1^{\prime} 02^{\prime \prime}$ \\
\hline$\Delta \theta$ Z (roll) & $+1^{\prime \prime}$ \\
\hline
\end{tabular}

Residual misalignment between optical and mechanical axes 


\section{CHANNELS CO-ALIGNMENT}

Each channel

- interferometrically aligned

- co-aligned wrt reference cube.

The Instrument Line of sight (ILS) = average of the two HRI channels LoS.

The ILS offset vs. UARF (front reference cube) and vs. URF (mechanical axis) 


\section{Conclusions}

- The 3 telescopes have been aligned interferometrically and are aligned wrt to a reference cube

- Verification of the spectral performances, carried out during the end-to-end calibration, allowed us to validate the complete acquisition chain (mirrors, filters, and detectors to CEB data processing).

- The EUI instrument is now installed on-board the Solar Orbiter spacecraft, co-aligned with the other remote-sensing instruments (SPICE, PHI, Metis, STIX), in preparation for the satellite environmental tests

- Launch planned in 2020. 


\section{Conclusions}

\section{Thank you for your attention}

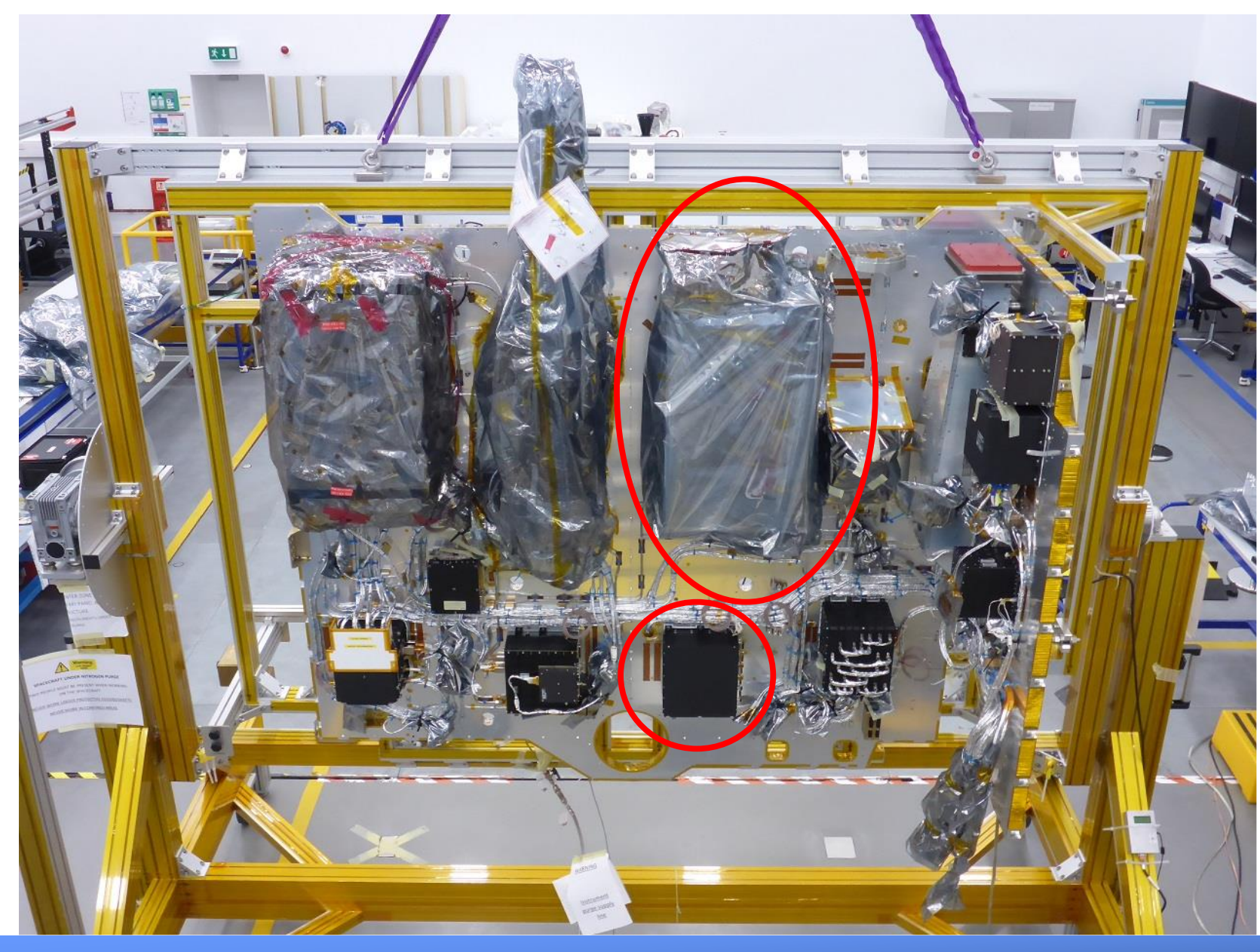

